\title{
BMJ Open Gender differences in the accuracy of dietary assessment methods to measure energy intake in adults: protocol for a systematic review and meta-analysis
}

To cite: McKenzie BL, Coyle DH, Burrows T, et al. Gender differences in the accuracy of dietary assessment methods to measure energy intake in adults: protocol for a systematic review and meta-analysis. BMJ Open 2020;10:e035611. doi:10.1136/ bmjopen-2019-035611

- Prepublication history and additional material for this paper are available online. To view these files, please visit the journal online (http://dx.doi. org/10.1136/bmjopen-2019035611).

Received 07 November 2019 Revised 30 March 2020 Accepted 13 May 2020

D Check for updates

(c) Author(s) (or their employer(s)) 2020. Re-use permitted under CC BY-NC. No commercial re-use. See rights and permissions. Published by BMJ.

For numbered affiliations see end of article.

\section{Correspondence to} Briar L McKenzie; bmckenzie@georgeinstitute. org.au

\section{ABSTRACT}

Introduction Diet is an important modifiable risk factor for many chronic diseases. Measurement of dietary intake usually relies on self-report, subject to multiple biases. There is a need to understand gender differences in the self-report of dietary intake and the implications of any differences in targeting nutrition interventions. Literature in this area is limited and it is currently unknown whether self-report dietary assessment methods are equally accurate for women and men. The aim of this systematic review is to determine whether there are differences by gender in reporting energy intake compared with a reference measure of total energy expenditure.

Methods and analysis A comprehensive search of published original research studies will be performed in MEDLINE, Scopus, Web of Science, EMBASE, CINAHL and Cochrane library. Original research studies will be included if they were conducted in free-living/unhospitalised adults and included a measure for both women and men of (a) self-reported energy intake and (b) total energy expenditure by doubly labelled water. One author will conduct the electronic database searches, two authors will independently screen studies, conduct a quality appraisal of the included studies using standardised tools and extract data. If further information is needed, then study authors will be contacted. If appropriate, a random-effects meta-analysis will be conducted, with inverse probability weighting, to quantify differences in the mean difference in agreement between reported energy intake and measured energy expenditure between women and men, by selfreport assessment method. Subgroup analyses will be conducted by participant factors, geographical factors and study quality.

Ethics and dissemination All data used will be from published primary research studies or deidentified results provided at the discretion of any study authors that we contact. We will submit our findings to a peer-reviewed scientific journal and will disseminate results through presentations at international scientific conferences. PROSPERO registration number CRD42019131715.

\section{INTRODUCTION}

The burden of nutrition-related disease and disease risk factors is increasing for women
Strengths and limitations of this study

- To the best of our knowledge, this systematic review will be the first to investigate gender differences in the accuracy of self-reported energy intake in comparison with a reference measure of energy expenditure, doubly labelled water.

- If appropriate, we will meta-analyse the difference in mean differences in the accuracy of self-reported energy intake, in comparison with energy expenditure as measured by doubly labelled water, by gender in order to quantify differences between women and men.

- Comparable studies, with data disaggregated into woman/man categories, may be limited.

- We are only including studies published in the English language, which may lead to language bias.

and men globally. ${ }^{1}$ Approximately a quarter of deaths were estimated to be attributable to poor diets in 2017, ${ }^{1}$ therefore, monitoring of dietary intakes at a population level is crucial for the targeting of interventions. Nutrition epidemiology has been criticised in relation to the use of self-reported diet measures, subject to multiple biases, including misreporting. ${ }^{2}$ Commonly used self-reported diet measures include 24-hour diet recall, diet histories, food records and food frequency questionnaires. These measures enable the assessment of dietary intakes at the individual and/or group level and provide information about eating habits, nutrient intakes (eg, energy and fibre) and micronutrient intakes (eg, sodium). Doubly labelled water is an objective reference measure of total energy expenditure, based on providing participants with water in which the hydrogen and oxygen have been replaced with uncommon isotopes that can be measured in urine. ${ }^{2}$ In weight-stable conditions, energy expenditure correlates to 
energy intake. However, measurement of doubly labelled water is expensive and holds a high respondent burden and is therefore not routinely conducted as part of surveys.

Over the past decade, a growing body of high-quality research has identified differing impacts of noncommunicable disease risk factors, such as high systolic blood pressure, diabetes and smoking, on cardiovascular disease outcomes, for women and men. ${ }^{34}$ However, dietary intake as a risk factor for disease outcomes has not been investigated to this extent via a gender lens. Although there is evidence that self-reported dietary behaviours and intake differ for women and men, ${ }^{5-8}$ it is unclear whether these are real differences or due to systematic misreporting of intake by women and men. Given the Sustainable Development Goals (SDG) of achieving good health and well-being (SDG 3) and gender equality (SDG $5),{ }^{9}$ it is important to investigate gender differences in dietary intake and any relationship with health outcomes to inform nutrition interventions.

In order to investigate gender differences in dietary intake, we first need to know if there is a differential reporting bias of dietary intake between women and men. This current review, to the best of our knowledge, will be the first to systematically review studies that have investigated dietary intake via self-reported measures compared with doubly labelled water, disaggregated for women and men. If a meta-analysis is possible, then it will also be the first to quantify gender differences between energy intake from self-reported dietary assessment methods and energy expenditure.

\section{OBJECTIVE}

The objective is to conduct a systematic review and metaanalysis comparing energy intake assessed using selfreported dietary assessment methods with measured energy expenditure for women and men.

\section{METHODS}

\section{Terminology: gender/sex}

According to the WHO, gender refers to 'the socially constructed characteristics of women and men such as norms, roles and relationships of, and between, groups of women and men. While most people are born either male or female, they are taught appropriate norms and behaviours-including how they should interact with others of the same or opposite sex within households, communities and workplaces'. ${ }^{10}$ In comparison, sex is 'the classification of living things, as male or female according to their reproductive organs and functions assigned by chromosomal complement'. ${ }^{11}$ In relation to our study, we are likely to include studies with data disaggregated by sex (a binary male/female measure). However, given the reference measure of doubly labelled water gives a value of energy expenditure at a constant for men and women, we hypothesise that any differences observed in the accuracy of self-reported measures are due to genderbased reasons; as such, the term gender (woman/man) has been used throughout this protocol.

\section{Protocol registration and review reporting}

This systematic review has been registered with the International Prospective Register of Systematic Reviews (PROSPERO).${ }^{12}$ We used the Preferred Reporting Items for Systematic reviews and Meta-Analysis Protocols (PRISMA-P) checklist when writing this protocol ${ }^{13}$ and we will conduct this systematic review in line with the PRISMA guidelines. $^{14}$

\section{Data sources and searches}

An electronic literature search will be conducted using the following databases: MEDLINE, Scopus, Web of Science, EMBASE, CINAHL and Cochrane Central Register of Controlled Trials. All peer-reviewed original research articles published before March 2020 will be included. The reference list of included studies will be searched for further relevant studies. Combinations of key words (diet*, nutrition, self, survey, diet*survey, diet*questionnaire, diet*recall, diet*record, food recall and doubly labelled water) and subject headings (diet, eating, energy intake, nutrition assessment, dietary intake, diet assessment, energy expenditure, surveys and questionnaires, self-report and diet surveys) will be used in the respective databases. The search strategy was designed in consultation with the University of New South Wales librarian services and trialled by two authors. The electronic database searches will be conducted by one author (BLM). See the online supplementary table 1 for an example of the MEDLINE search strategy.

\section{Study screening}

Screening of studies will be conducted based on the following inclusion and exclusion criteria and will be conducted in Covidence, ${ }^{15}$ an online systematic review data management software.

\section{Inclusion criteria}

- Published original research studies, in peer-reviewed journals.

- Studies conducted in free-living/un-hospitalised adults aged 18 years or older.

- Studies that include a measure of self-reported energy intake and a measure of total energy expenditure via doubly labelled water.

- Studies that include at least two participants of each gender and that present results disaggregated by gender.

- The full text is available in English.

\section{Exclusion criteria}

- Studies conducted in populations where significant weight change is likely. For example, conducted in elite athletes, weight loss trials or in people with medical conditions where weight change is a common side effect of the disease and/or treatment. 
- Studies conducted in hospitalised populations, as these populations are unlikely to be eating in their usual manner and/or are unlikely to have control over their food choices.

- Controlled feeding studies.

- Published conference abstracts.

- Published study protocols.

- Published reviews.

- Studies conducted on animals.

\section{Notes on inclusion/exclusion criteria}

Reviews will be excluded, however, their reference lists will be searched for studies. Studies will be excluded if results are not disaggregated by male and female (man/ woman) categories. If some results are presented in a disaggregated manner but we require more information, then we will contact the authors of the studies. For publications that have used information from the same study population, findings from the first (earliest) publication that meet our inclusion and exclusion criteria will be included.

\section{Study selection}

The title and abstracts of the identified studies from the electronic searches will be screened by two authors (BLM and DHC) to assess potential eligibility. Full texts of the potentially eligible studies will then be retrieved and reviewed against the inclusion and exclusion criteria in order to obtain our final sample of studies, again in duplicate. Any disagreement in eligibility of studies will be discussed at both the title and abstract and the fulltext review stage, and a third author (ER) will be included in discussions if necessary. We will present the studies included and excluded at each stage of the screening process in a PRISMA flowchart. ${ }^{14}$ For the full-text review stage, we will also provide reasons for the exclusion of studies.

\section{Data extraction and management}

Relevant variables will be extracted using a data extraction template (Microsoft Excel). This template will be piloted by two authors (BLM and DHC) on a subsample of the full texts before the commencement of data extraction and will be discussed with the author team. Data will be extracted independently by two authors (BLM and DHC) and cross-checked, with any disagreements resolved by discussion with a third author (ER) when consensus cannot be achieved. Data to be extracted will include: author, title, journal, year of publication, study setting, study design, study population, sample size, aim, participant characteristics (including any weight change during the study and prevalence of chronic disease states), method(s) used to measure dietary intake, methods used for energy expenditure (period of doubly labelled water collection, number of samples and dosage of labelled water given), intervention details (where applicable), study outcomes (reported mean energy intake and energy expenditure, any reported correlations between energy intake and expenditure, limits of agreement and percentage under, accurate and over reporters) and funding source. Gender-disaggregated data will be extracted for all variables if possible.

\section{Quality assessment of included studies}

Our final pool of studies will be assessed for quality using The Academy of Nutrition and Dietetics evidence analysis manual: steps in the academy evidence analysis process, ${ }^{16}$ which includes a Quality Criteria Checklist. This checklist includes categories with questions regarding the relevance of the study to clinical (dietetic) practice and the validity of the research. The questions regarding study validity cover study sampling, blinding of interventions, reliability of outcome measurement, statistical analysis and the likely influence of study funding or sponsorship. ${ }^{16}$ Each category is marked positive, negative or neutral, and an overall assessment is made depending on the number of categories and which specific categories are answered in a particular way. A level of evidence will also be defined, following the National Health and Medical Research Council levels of evidence and grades for developers' guidelines. ${ }^{17}$ The quality of each included study will be assessed by two researchers (BLM and DHC) independently, and any disagreements will be resolved by discussion with a third author (ER).

\section{Data synthesis, assessing heterogeneity and publication bias}

If two or more studies of a similar methodology are identified in our review, we will quantify gender differences in the agreement between reported energy intake and measured energy expenditure by a random-effects metaanalysis model with inverse variance weighting. We will extract the mean values of energy intake and energy expenditure, with corresponding measures of variability, by gender. The values of energy intake and energy expenditure will be extracted in kilojoules per day. If data were reported in kilocalories, then they will be converted to kilojoules by multiplying by $4.184 .^{2}$ The mean difference and $95 \% \mathrm{CI}$ between intake and expenditure will then be calculated by gender. In order to quantify gender differences, the difference in the mean differences will be calculated within each study and pooled across studies in the meta-analysis with corresponding $95 \%$ CI. ${ }^{4}$ Separate meta-analyses will be conducted for each self-reported dietary assessment method (24-hour dietary recall, diet histories, food records and food frequency questionnaires). Heterogeneity will be assessed using Cochran's Q-test and the $\mathrm{I}^{2}$ statistic. Subgroup analyses will be conducted by participant factors (age, weight, education and chronic disease states), geographical factors (setting (urban/rural), country income level and world region) and by study quality assessment. The Grading of Recommendations Assessment, Development, and Evaluation (GRADE) guidelines will be followed when developing our tables to display our results. ${ }^{18}$ The analysis will be conducted using STATA V.15 statistical software. 
The narrative synthesis of the included studies will be conducted, where all studies will be summarised, including findings from studies that are not able to be included in the meta-analysis.

\section{PATIENT AND PUBLIC INVOLVEMENT}

Patients and public were not involved in the design of the systematic review protocol. Their involvement is not applicable given that no participant recruitment will take place for this review.

\section{ETHICS AND DISSEMINATION}

We are not collecting primary data and will only be using published or author provided (deidentified) data, therefore, ethical clearance is not needed. We will publish this review and meta-analysis in a recognised peer-reviewed public health nutrition journal under open access. We will also present our findings at an international scientific conference.

\section{Author affiliations}

${ }^{1}$ The George Institute for Global Health, University of New South Wales, Sydney, New South Wales, Australia

${ }^{2}$ School of Health Sciences, Faculty of Health and Medicine, The University of Newcastle, Callaghan, New South Wales, Australia

${ }^{3}$ The George Institute for Global Health, University of Oxford, Oxford, UK

${ }^{4}$ Julius Center for Health Sciences and Primary Care, University Medical Center Utrecht, Utrecht, The Netherlands

${ }^{5}$ Department of Epidemiology, Johns Hopkins University, Baltimore, Maryland, USA ${ }^{6}$ Department of Global Health and Population, Harvard T.H. Chan School of Public Health, Boston, Massachusetts, USA

\section{Twitter Briar L McKenzie @briarlou}

Acknowledgements The University of New South Wales Academic Engagement Librarians, for their help in developing the search strategy.

Contributors BLM, DHC and JW conceptualised and designed the protocol. BLM drafted the initial manuscript. Search terms were drafted in consultation with a Librarian at the University of New South Wales. Data extraction processes and statistical analysis were drafted by BLM, then reviewed and discussed with all authors. All authors provided critical insights: TB, CEC and ER provided expert content knowledge on energy intake and energy expenditure methods; CC, SAEP and MW provided insight into the proposed approach to quantitative synthesis of the data; and RN, LMJ and JW provided general oversight. All authors contributed to the manuscript and approved the final written manuscript.

Funding BLM is supported by a UNSW Scientia PhD scholarship for her PhD titled "investigating gender differences in dietary intake and behaviours and their relationship with cardio-metabolic disease".

\section{Competing interests None declared.}

Patient and public involvement Patients and/or the public were not involved in the design, or conduct, or reporting, or dissemination plans of this research.

Patient consent for publication Not required.

Provenance and peer review Not commissioned; externally peer reviewed.
Open access This is an open access article distributed in accordance with the Creative Commons Attribution Non Commercial (CC BY-NC 4.0) license, which permits others to distribute, remix, adapt, build upon this work non-commercially, and license their derivative works on different terms, provided the original work is properly cited, appropriate credit is given, any changes made indicated, and the use is non-commercial. See: http://creativecommons.org/licenses/by-nc/4.0/.

ORCID iDs

Briar L McKenzie http://orcid.org/0000-0001-6972-6617

Emalie Rosewarne http://orcid.org/0000-0001-5748-3953

\section{REFERENCES}

1 Afshin A, Sur PJ, Fay KA, et al. Health effects of dietary risks in 195 countries, 1990-2017: a systematic analysis for the global burden of disease study 2017. Lancet 2019;393:1958-72.

2 Gibson RS. Principles of nutritional assessment. USA: Oxford university press, 2005.

3 Millett ERC, Peters SAE, Woodward M. Sex differences in risk factors for myocardial infarction: cohort study of UK Biobank participants. BMJ 2018;363:k4247.

4 Woodward M. Rationale and tutorial for analysing and reporting sex differences in cardiovascular associations. Heart 2019;105:heartjnl-2019-315299:1701-8.

5 McKenzie B, Santos JA, Trieu K, et al. The science of salt: a focused review on salt-related knowledge, attitudes and behaviors, and gender differences. J Clin Hypertens 2018;20:850-66.

6 Arganini C, Saba A, Comitato R, et al. Gender differences in food choice and dietary intake in modern Western societies. In: Public health-social and behavioral health. China: InTech, 2012.

7 Bennett E, Peters SAE, Woodward M. Sex differences in macronutrient intake and adherence to dietary recommendations: findings from the UK Biobank. BMJ Open 2018;8:e020017.

8 Marino M, Masella R, Bulzomi P, et al. Nutrition and human health from a sex-gender perspective. Mol Aspects Med 2011;32:1-70.

9 The United Nations. The sustainable development goals 2015, 2019. Available: https://www.un.org/sustainabledevelopment/sustainabledevelopment-goals/

10 World Health Organization. Gender, equity and human rights, 2019. Available: https://www.who.int/gender-equity-rights/understanding/ gender-definition/en/

11 National Research Council, Institute of Medicine, Committee on Understanding the Biology of Sex and Gender Differences. Exploring the biological contributions to human health: does sex matter. Washington, DC: The National Academies Press, 2001.

12 McKenzie B, Coyle D, Sparks E, et al. Sex/gender differences in the validity of dietary assessment methods to measure energy intake in adults: a systematic review PROSPERO International prospective register of systematic reviews National Institute for health research, 2019. Available: https://www.crd.york.ac.uk/prospero/display_record. php?RecordID $=131715$

13 Moher D, Shamseer L, Clarke M, et al. Preferred reporting items for systematic review and meta-analysis protocols (PRISMA-P) 2015 statement. Syst Rev 2015;4:1.

14 Moher D, Liberati A, Tetzlaff J, et al. Preferred reporting items for systematic reviews and meta-analyses: the PRISMA statement. Ann Intern Med 2009;151:264-9.

15 Covidence. Covidence World-class systematic review management, 2019. Available: https://www.covidence.org/home

16 Academy of Nutrition and Dietetics, Research International and Strategic Business Development Team. Evidence analysis manual: steps in the Academy evidence analysis process. Chicago, 2016.

17 The National Health and Medical Research Council. NHMRC additional levels of evidence and grades for recommendations for developers of guidelines. Canberra: The National Health and Medical Research Council, Australian Government, 2009.

18 Guyatt GH, Oxman AD, Santesso N, et al. GRADE guidelines: 12. preparing summary of findings tables-binary outcomes. J Clin Epidemiol 2013;66:158-72. 